\title{
New mathematics for the nonadditive Tsallis' scenario
}

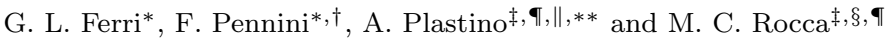 \\ *Facultad de Ciencias Exactas y Naturales, \\ Universidad Nacional de La Pampa, \\ Peru 151, 6300 Santa Rosa, La Pampa, Argentina \\ Uruguay 151, Santa Rosa, La Pampa, Argentina \\ ${ }^{\dagger}$ Universidad Católica del Norte, \\ Av. Angamos 0610, Antofagasta, Chile \\ ${ }^{\ddagger}$ Departamento de Física, \\ Universidad Nacional de La Plata, Argentina \\ $\S$ Departamento de Matemática, \\ Universidad Nacional de La Plata, Argentina \\ "Consejo Nacional de Investigaciones Científicas y Tecnológicas \\ (IFLP-CCT-CONICET)-C. C. 727, 1900 La Plata, Argentina \\ "SThAR - EPFL, Lausanne, Switzerland \\ **angeloplastino@gmail.com
}

Received 5 January 2017

Accepted 1 March 2017

Published 4 April 2017

\begin{abstract}
In this paper, we investigate quantum uncertainties in a Tsallis' nonadditive scenario. To such an end we appeal to $q$-exponentials (qEs), that are the cornerstone of Tsallis' theory. In this respect, it is found that some new mathematics is needed and we are led to construct a set of novel special states that are the $\mathrm{qE}$ equivalents of the ordinary coherent states (CS) of the harmonic oscillator (HO). We then characterize these new Tsallis' special states by obtaining the associated (i) probability distributions (PDs) for a state of momentum $k$, (ii) mean values for some functions of space an momenta and (iii) concomitant quantum uncertainties. The latter are then compared to the usual ones.
\end{abstract}

Keywords: Tsallis' statistics; quantum uncertainties; q-exponentials.

PACS numbers: 05.30.-d, 05.20.-y, 05.70.-a

\section{Introduction}

During more than 25 years, an important topic in statistical mechanics theory revolved around the notion of generalized nonadditive statistics, pioneered by Tsallis. 1 It has been amply demonstrated that, on many occasions, the celebrated Boltzmann-Gibbs logarithmic entropy does not yield a correct description of the system under scrutiny. $\frac{2}{}$ Other entropic forms, called nonadditive entropies $S_{q}$

\footnotetext{
** Corresponding author.
} 
$(q \in \mathcal{R})$, produce a much better performance. $\stackrel{2}{ }$ The nonadditive law reads, for two independent systems $A$ and $B, S_{q}(A B)=S(A)+S(B)+(1-q) S(A) S(B)$. One may cite a large number of such instances, for example, nonergodic systems exhibiting a complex dynamics. $\underline{2}$

The nonextensive statistical mechanics of Tsallis' has been employed to fruitfully discuss phenomena in variegated fields. One may mention, for instance, high-energy physics,,$\frac{3,4}{4}$ spin-glasses,$\frac{5}{-}$ cold atoms in optical lattices,,$\underline{6}$ trapped ions,,$\frac{7}{}$ anomalous diffusion,$\frac{8,9}{9}$ dusty plasmas, $\frac{10}{-}$ low-dimensional dissipative and conservative maps in dynamical systems,,$\underline{11}-\underline{13}$ turbulent flows, $\underline{14}$ Levy flights, $\frac{16}{2}$ the QCD-based Nambu, Jona, Lasinio model of a many-body field theory, $\frac{17}{1}$ etc. Notions related to $q$ statistical mechanics have been found useful not only in physics but also in chemistry, biology, mathematics, economics, informatics and quantum mechanics $\underline{18} \underline{-21}$ Given the importance of the Tsallis-materials, the associated mathematics acquires particular relevance. We believe to be here making some interesting contributions to such mathematics.

The probability distribution (PD) associated to the nonadditive, $q$-statistics is the so-called $q$-exponential, $\underline{2}$ that becomes the customary exponential $(\mathrm{CE})$ in the limit $q \rightarrow 1$. Physical states described via qEs are the focus of our present concerns. We obtain them by replacing CEs by (qEs) whenever physical states expressed in CE-terms emerge. A reference to coherent states (CS) is then needed (see, for instance, Ref. 15). Then, with regard to the line of inquiry just mentioned, we

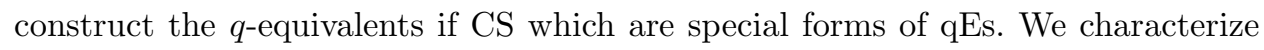
the ensuing $q$-equivalents by evaluation of its main properties, and then discuss the associated quantum uncertainties. A note of warning is due here. Our new $q$ equivalents have nothing to do with the so-called q-deformed CS of Quesne, EreminMeldianov, and others. These are CS of a deformed harmonic oscillator (HO). $\underline{22}$

\section{Prerequisites}

Let us briefly remind the reader of the CS of the HO $|\alpha\rangle$, or Glauber states. $\underline{23} \underline{25}$ A CS $|\alpha\rangle$ is a specific kind of quantum state of minimum uncertainty, the one that most resembles a classical state. It is applicable to the quantum HO, the electromagnetic field, etc., and describes a maximal kind of coherence and a classical kind of behavior. The states $|\alpha\rangle$ are normalized, i.e., $\langle\alpha \mid \alpha\rangle=1$, and they provide us with a resolution of the identity operator

$$
\int \frac{\mathrm{d}^{2} \alpha}{\pi}|\alpha\rangle\langle\alpha|=1,
$$

which is a completeness relation for the CS. $\underline{25}$ The standard CS $|\alpha\rangle$ for the HO are eigenstates of the annihilation operator $\hat{a}$, with complex eigenvalues

$$
\alpha=\frac{q+i p}{\sqrt{2}}
$$

which satisfy $\hat{a}|\alpha\rangle=\alpha|\alpha\rangle . \underline{25}$ 
The $n$th $\mathrm{HO}$ eigenfunction is

$$
\phi_{n}(x)=\left(\frac{m \omega}{\hbar}\right)^{\frac{1}{4}} \mathcal{H}_{n}\left(\sqrt{\frac{m \omega}{\hbar}} x\right)
$$

where $\mathcal{H}_{n}$ is Hermite's $n$th order generalized function

$$
\mathcal{H}_{n}(x)=\left(\pi^{\frac{1}{2}} 2^{n} n !\right)^{-\frac{1}{2}} e^{-\frac{x^{2}}{2}} H_{n}(x)
$$

while $H_{n}$ is the concomitant Hermite polynomial. In the $x$-representation, the CS reads

$$
\psi_{\alpha}(x)=e^{-\frac{|\alpha|^{2}}{2}} \sum_{n=0}^{\infty} \frac{\alpha^{n}}{\sqrt{n !}} \phi_{n}(x)
$$

or

$$
\psi_{\alpha}(x)=\left(\frac{m \omega}{\hbar}\right)^{\frac{1}{4}} e^{-\frac{|\alpha|^{2}}{2}} \sum_{n=0}^{\infty} \frac{\alpha^{n}}{\sqrt{n !}} \mathcal{H}_{n}\left(\sqrt{\frac{m \omega}{\hbar}} x\right) .
$$

For convenience, we choose $\sqrt{\frac{m \omega}{\hbar}}=1$. Thus, for the HO, we have

$$
\phi_{n}(x)=\mathcal{H}_{n}(x)
$$

and for its CS

$$
\psi_{\alpha}(x)=e^{-\frac{|\alpha|^{2}}{2}} \sum_{n=0}^{\infty} \frac{\alpha^{n}}{\sqrt{n !}} \mathcal{H}_{n}(x) .
$$

We use at this point the interesting fact that the CS can be made to compactly read (see Appendix A)

$$
\psi_{\alpha}(x)=\pi^{-\frac{1}{4}} e^{-\frac{\alpha^{2}}{2}} e^{-\frac{|\alpha|^{2}}{2}} e^{-\frac{x^{2}}{2}} e^{\sqrt{2} \alpha x} .
$$

To prove that $\underline{(2.9)}$ is equal to $\underline{(2.8)}$, we expand $\underline{(2.9)}$ à la Hermite

$$
\psi_{\alpha}(x)=\sum_{n=0}^{\infty} a_{n} \mathcal{H}_{n}(x)
$$

and compute $a_{n}$ as

$$
a_{n}=\int_{-\infty}^{\infty} \psi_{\alpha}(x) \mathcal{H}_{n}(x) d x
$$

Accordingly,

$$
a_{n}=\pi^{-\frac{1}{4}} e^{-\frac{\alpha^{2}}{2}} e^{-\frac{|\alpha|^{2}}{2}} \int_{-\infty}^{\infty} e^{-\frac{x^{2}}{2}} e^{\sqrt{2} \alpha x} \mathcal{H}_{n}(x) d x
$$

that can be recast as

$$
a_{n}=\frac{\pi^{-\frac{1}{4}} e^{-\frac{|\alpha|^{2}}{2}}}{\left(n ! 2^{n} \pi^{\frac{1}{2}}\right)^{\frac{1}{2}}} \int_{-\infty}^{\infty} e^{-\left(x-\frac{\alpha}{\sqrt{2}}\right)^{2}} H_{n}(x) d x .
$$


We apply now an Integral-Table result (see Ref. 27) to obtain

$$
a_{n}=\frac{\pi^{-\frac{1}{4}} e^{-\frac{|\alpha|^{2}}{2}}}{\left(n ! 2^{n} \pi^{\frac{1}{2}}\right)^{\frac{1}{2}}} \pi^{\frac{1}{2}} 2^{\frac{n}{2}} \alpha^{n}
$$

or

$$
a_{n}=(n !)^{-\frac{1}{2}} \alpha^{n} e^{-\frac{|\alpha|^{2}}{2}} .
$$

Replacing now $(\underline{2.15})$ into $(\underline{2.10})$, we reach $\underline{(2.8)}$ and prove $(2.9)$. Our results in this paper are based on Eq. (2.9), translated into $q$-parlance.

\section{Special States Associated to the Nonadditive, $q$-Statistics}

We start here work in this respect, and wish to report some advances. An extremely important and critical result is (2.9) for an ordinary CS, that we will $q$-generalize via replacement $\mathrm{CE} \rightarrow \mathrm{qE}$. The ensuing state, that one may call a Tsallis' pseudocoherent one, is obtained, we reiterate, by replacing the exponential $(2.9)$ by the associated $\mathrm{qE} e_{q}(x)^{2}$

$$
e_{q}(x)=[1+(1-q) x]^{1 / 1-q} ; \quad q \in \mathcal{R},
$$

that becomes the ordinary exponential at $q=1$. Accordingly, we have

$$
\psi_{\alpha q}(x)=A(q, \alpha)\left[1+\frac{q-1}{2}\left(x^{2}-2 \sqrt{2} \alpha x+|\alpha|^{2}+\alpha^{2}\right)\right]^{\frac{1}{1-q}},
$$

where $A(q, \alpha)$ is a normalization constant to be determined. Remember that these states have nothing to do with the so-called q-CS of Quesne, Eremin-Meldianov and others. $\underline{22}$

We proceed now to determine the mathematical apparatus associated to these states $\psi_{\alpha q}$, i.e., (1) normalization, (2) overlaps, (3) PDs (4) mean values and (5) uncertainties, in order to describe the nature of our special states, which is the goal of this paper.

We need to apply some cumbersome mathematics. In particular, Lauricella functions $F_{D}$, described in Appendix B, become of essence. They are extensions to several variables of the hypergeometric functions.

\subsection{Normalization}

For our present work on new $q$-states we need, first of all, an explicit expression for the overlap involved in the normalization process

$$
\begin{aligned}
\left\langle\psi_{\alpha q} \mid \psi_{\alpha q}\right\rangle= & A^{2}(q, \alpha) \int_{-\infty}^{\infty}\left[1+\frac{q-1}{2}\left(x^{2}-2 \sqrt{2} \alpha x+|\alpha|^{2}+\alpha^{2}\right)\right]^{\frac{1}{1-q}} \\
& \otimes\left[1+\frac{q-1}{2}\left(x^{2}-2 \sqrt{2} \alpha^{*} x+|\alpha|^{2}+\alpha^{* 2}\right)\right]^{\frac{1}{1-q}} d x
\end{aligned}
$$


This necessitates applying the Lauricella functions $F_{D}$. We recast (3.3) in the form

$$
\begin{aligned}
\left\langle\psi_{\alpha q} \mid \psi_{\alpha q}\right\rangle= & A^{2}(q, \alpha)\left(\frac{q-1}{2}\right)^{\frac{2}{1-q}} \int_{-\infty}^{\infty}\left(x-\sqrt{2} \alpha-\sqrt{|\alpha|^{2}+\alpha^{2}-\frac{2}{q-1}}\right)^{\frac{1}{1-q}} \\
& \otimes\left(x-\sqrt{2} \alpha+\sqrt{|\alpha|^{2}+\alpha^{2}-\frac{2}{q-1}}\right)^{\frac{1}{1-q}} \\
& \otimes\left(x-\sqrt{2} \alpha^{*}-\sqrt{|\alpha|^{2}+\alpha^{* 2}-\frac{2}{q-1}}\right)^{\frac{1}{1-q}} \\
& \otimes\left(x-\sqrt{2} \alpha^{*}+\sqrt{|\alpha|^{2}+\alpha^{* 2}-\frac{2}{q-1}}\right)^{\frac{1}{1-q}} d x
\end{aligned}
$$

Utilizing Eq. (B.3) from Appendix B, we find

$$
\begin{aligned}
\left\langle\psi_{\alpha q} \mid \psi_{\alpha q}\right\rangle & =A^{2}(q, \alpha) \frac{q-1}{5-q}\left(\frac{q-1}{2}\right)^{\frac{2}{1-q}} \otimes F_{D}\left(\frac{5-q}{q-1} ; \frac{1}{q-1}, \frac{1}{q-1}, \frac{1}{q-1}, \frac{1}{q-1}, \frac{4}{q-1}\right. \\
& 1+\sqrt{2} \alpha+\sqrt{\alpha^{2}-|\alpha|^{2}-\frac{2}{q-1}}, 1+\sqrt{2} \alpha-\sqrt{\alpha^{2}-|\alpha|^{2}-\frac{2}{q-1}} \\
& \left.1+\sqrt{2} \alpha^{*}+\sqrt{\alpha^{* 2}-|\alpha|^{2}-\frac{2}{q-1}}, 1+\sqrt{2} \alpha^{*}-\sqrt{\alpha^{* 2}-|\alpha|^{2}-\frac{2}{q-1}}\right) .
\end{aligned}
$$

Now, because of the normalization requirement

$$
\left\langle\psi_{\alpha q} \mid \psi_{\alpha q}\right\rangle=1
$$

we get for the constant $A(q, \alpha)$ the expression

$$
\begin{aligned}
A(q, \alpha)= & {\left[\frac { q - 1 } { 5 - q } ( \frac { q - 1 } { 2 } ) ^ { \frac { 2 } { 1 - q } } F _ { D } \left(\frac{5-q}{q-1} ; \frac{1}{q-1}, \frac{1}{q-1}, \frac{1}{q-1}, \frac{1}{q-1}, \frac{4}{q-1}\right.\right.} \\
& 1+\sqrt{2} \alpha+\sqrt{\alpha^{2}-|\alpha|^{2}-\frac{2}{q-1}}, 1+\sqrt{2} \alpha-\sqrt{\alpha^{2}-|\alpha|^{2}-\frac{2}{q-1}} \\
& 1+\sqrt{2} \alpha^{*}+\sqrt{\alpha^{* 2}-|\alpha|^{2}-\frac{2}{q-1}}, \\
& \left.\left.1+\sqrt{2} \alpha^{*}-\sqrt{\alpha^{* 2}-|\alpha|^{2}-\frac{2}{q-1}}\right)\right]^{-\frac{1}{2}}
\end{aligned}
$$

\subsection{Scalar product}

Usual CS are not orthogonal. Again, we will apply the Lauricella functions $F_{D}$ (Appendix B). Thus, we compute now the scalar product (overlap) of two arbitrary 
G. L. Ferri et al.

states $\psi_{\alpha q}$

$$
\begin{aligned}
& \left\langle\psi_{\alpha q} \mid \psi_{\beta q}\right\rangle \\
& =A(q, \alpha) A(q, \beta) \frac{q-1}{5-q}\left(\frac{q-1}{2}\right)^{\frac{2}{1-q}} \\
& \otimes F_{D}\left(\frac{5-q}{q-1} ; \frac{1}{q-1}, \frac{1}{q-1}, \frac{1}{q-1}, \frac{1}{q-1}, \frac{4}{q-1} ;\right. \\
& 1+\sqrt{2} \alpha+\sqrt{\alpha^{2}-|\alpha|^{2}-\frac{2}{q-1}}, 1+\sqrt{2} \alpha-\sqrt{\alpha^{2}-|\alpha|^{2}-\frac{2}{q-1}}, \\
& \left.1+\sqrt{2} \beta^{*}+\sqrt{\beta^{* 2}-|\beta|^{2}-\frac{2}{q-1}}, 1+\sqrt{2} \beta^{*}-\sqrt{\beta^{* 2}-|\beta|^{2}-\frac{2}{q-1}}\right) .
\end{aligned}
$$

The nonnormalized Tsallis' pseudo-CS

$$
\phi_{\alpha q}(x)=\frac{\psi_{\alpha q}(x)}{A(q, \alpha)}
$$

is a proper vector corresponding to the proper value $\alpha$ of the operator $a_{q}$ given by

$$
a_{q}(x) f(x)=\frac{x}{\sqrt{2}} f(x)+\frac{f^{1-q}(x)}{\sqrt{2}} \frac{d f(x)}{d x} .
$$

Note when $q=1, a_{q}$ is the usual annihilation operator of the HO.

\subsection{Associated probability distribution (PD)}

We pass now to the PD associated to a Tsallis pseudo-CS. We start by noting that

$$
|\alpha, q\rangle=A(q, \alpha) \int_{-\infty}^{\infty}\left[1+\frac{q-1}{2}\left(x^{2}-2 \sqrt{2} \alpha x+|\alpha|^{2}+\alpha^{2}\right)\right]^{\frac{1}{1-q}}|x\rangle d x .
$$

Thus, the overlap between a plane wave of momentum $k$ and $|\alpha, q\rangle$ is

$$
\langle k \mid \alpha, q\rangle=\frac{A(q, \alpha)}{\sqrt{2 \pi}} \int_{-\infty}^{\infty} e^{-i k x}\left[1+\frac{q-1}{2}\left(x^{2}-2 \sqrt{2} \alpha x+|\alpha|^{2}+\alpha^{2}\right)\right]^{\frac{1}{1-q}} d x
$$

that can be rewritten as

$$
\begin{aligned}
\langle k \mid \alpha, q\rangle= & \frac{A(q, \alpha)}{\sqrt{2 \pi}} \int_{-\infty}^{\infty} e^{-i k x}\left[x+\sqrt{2} \alpha+\sqrt{\alpha^{2}-|\alpha|^{2}-\frac{2}{q-1}}\right]^{\frac{1}{1-q}} \\
\otimes & {\left[x+\sqrt{2} \alpha-\sqrt{\alpha^{2}-|\alpha|^{2}-\frac{2}{q-1}}\right]^{\frac{1}{1-q}} d x . }
\end{aligned}
$$


Using now the Integral-Table result $\frac{30}{}$ we find

$$
\begin{aligned}
\langle k \mid \alpha, q\rangle= & \frac{\operatorname{Sgn}(k) \sqrt{2 \pi} A(q, \alpha)|k|^{\frac{3-q}{q-1}}}{\Gamma\left(\frac{2}{q-1}\right)} e^{-\frac{i \pi \operatorname{Sgn}(k)}{q-1}} \otimes e^{i\left(\sqrt{2} \alpha+\sqrt{\alpha^{2}-|\alpha|^{2}-\frac{2}{q-1}}\right)} \phi \\
& \times\left(\frac{1}{q-1}, \frac{2}{q-1} ;-2 i \sqrt{\alpha^{2}-|\alpha|^{2}-\frac{2}{q-1}}|k|\right) .
\end{aligned}
$$

The PD we are looking for becomes

$$
\begin{aligned}
|\langle k \mid \alpha, q\rangle|^{2}= & \frac{2 \pi[A(q, \alpha)]^{2}|k|^{\frac{6-2 q}{q-1}}}{\left[\Gamma\left(\frac{2}{q-1}\right)\right]^{2}} \otimes e^{i\left[\sqrt{2}\left(\alpha-\alpha^{*}\right)+\sqrt{\alpha^{2}-|\alpha|^{2}-\frac{2}{q-1}}-\sqrt{\alpha^{* 2}-|\alpha|^{2}-\frac{2}{q-1}}\right]} \\
& \otimes \phi\left(\frac{1}{q-1}, \frac{2}{q-1} ;-2 i \sqrt{\alpha^{2}-|\alpha|^{2}-\frac{2}{q-1}}|k|\right) \\
& \otimes \phi\left(\frac{1}{q-1}, \frac{2}{q-1} ; 2 i \sqrt{\alpha^{* 2}-|\alpha|^{2}-\frac{2}{q-1}}|k|\right)
\end{aligned}
$$

and gives the probability of encountering momentum $k$ if the system is described by $|\alpha, q\rangle$.

\section{Towards Determining Uncertainties}

We need to evaluate several mean values to this end.

\subsection{Mean value of $x^{2}$}

We can calculate now $\left\langle x^{2}\right\rangle_{q}$. It is given by

$$
\begin{aligned}
\left\langle x^{2}\right\rangle_{q}= & A^{2}(q, \alpha)\left(\frac{q-1}{2}\right)^{\frac{2}{1-q}} \int_{-\infty}^{\infty}\left(x^{2}-2 \sqrt{\alpha^{*}} x+|\alpha|^{2}+\alpha^{* 2}+\frac{2}{q-1}\right)^{\frac{1}{1-q}} \\
& \otimes x^{2}\left(x^{2}-2 \sqrt{\alpha} x+|\alpha|^{2}+\alpha^{2}+\frac{2}{q-1}\right)^{\frac{1}{1-q}} d x
\end{aligned}
$$

Let $\beta_{1}, \beta_{2}, \beta_{3}, \beta_{4}$ be given by

$$
\begin{gathered}
\beta_{1}=\sqrt{2} \alpha^{*}+\sqrt{\alpha^{* 2}-|\alpha|^{2}-\frac{2}{q-1}}, \quad \beta_{2}=\sqrt{2} \alpha^{*}-\sqrt{\alpha^{* 2}-|\alpha|^{2}-\frac{2}{q-1}} \\
\beta_{3}=\sqrt{2} \alpha+\sqrt{\alpha^{2}-|\alpha|^{2}-\frac{2}{q-1}}, \quad \beta_{4}=\sqrt{2} \alpha-\sqrt{\alpha^{2}-|\alpha|^{2}-\frac{2}{q-1}} .
\end{gathered}
$$


G. L. Ferri et al.

Then we can write $\underline{(4.16)}$ as

$$
\begin{aligned}
\left\langle x^{2}\right\rangle_{q}= & A^{2}(q, \alpha)\left(\frac{q-1}{2}\right)^{\frac{2}{1-q}} \int_{-\infty}^{\infty} x^{2}\left(x-\beta_{1}\right)^{\frac{1}{1-q}}\left(x-\beta_{2}\right)^{\frac{1}{1-q}} \\
& \otimes\left(x-\beta_{3}\right)^{\frac{1}{1-q}}\left(x-\beta_{4}\right)^{\frac{1}{1-q}} d x .
\end{aligned}
$$

Applying now to $(\underline{B .3})$, and to Lauricella functions $F_{D}$, we obtain for $\underline{(4.18)}$

$$
\begin{aligned}
\left\langle x^{2}\right\rangle_{q}= & 2 A^{2}(q, \alpha)\left(\frac{q-1}{2}\right)^{\frac{2}{1-q}} \frac{\Gamma\left(\frac{7-3 q}{q-1}\right)}{\Gamma\left(\frac{4}{q-1}\right)} F_{D}\left(\frac{7-3 q}{q-1} ; \frac{1}{q-1}, \frac{1}{q-1}, \frac{1}{q-1}, \frac{1}{q-1}\right. \\
& \left.\frac{4}{q-1} ; 1+\beta_{1}, 1+\beta_{2}, 1+\beta_{3}, 1+\beta_{4}\right)
\end{aligned}
$$

\subsection{Mean value of $x$}

Once again, we apply here Lauricella functions $F_{D}$ (Appendix B). In the same way as above, we have for $\langle x\rangle_{q}$ the expression

$$
\begin{aligned}
\langle x\rangle_{q}= & A^{2}(q, \alpha)\left(\frac{q-1}{2}\right)^{\frac{2}{1-q}} \frac{\Gamma\left(\frac{6-2 q}{q-1}\right)}{\Gamma\left(\frac{4}{q-1}\right)} F_{D}\left(\frac{6-2 q}{q-1} ; \frac{1}{q-1}, \frac{1}{q-1}, \frac{1}{q-1}, \frac{1}{q-1}\right. \\
& \left.\frac{4}{q-1} ; 1+\beta_{1}, 1+\beta_{2}, 1+\beta_{3}, 1+\beta_{4}\right)
\end{aligned}
$$

\subsection{Mean value of $p^{2}$}

The evaluation of $\left\langle p^{2}\right\rangle_{q}$ is somewhat more involved. For it, we have

$$
\begin{aligned}
\left\langle p^{2}\right\rangle_{q}= & -A^{2}(q, \alpha)\left(\frac{q-1}{2}\right)^{\frac{2}{1-q}} \int_{-\infty}^{\infty}\left(x^{2}-2 \sqrt{\alpha^{*}} x+|\alpha|^{2}+\alpha^{* 2}+\frac{2}{q-1}\right)^{\frac{1}{1-q}} \\
& \otimes \frac{\partial^{2}}{\partial x^{2}}\left(x^{2}-2 \sqrt{\alpha} x+|\alpha|^{2}+\alpha^{2}+\frac{2}{q-1}\right)^{\frac{1}{1-q}} d x
\end{aligned}
$$

or

$$
\begin{aligned}
\left\langle p^{2}\right\rangle_{q}= & -\frac{A^{2}(q, \alpha)}{(1-q)}\left(\frac{q-1}{2}\right)^{\frac{2}{1-q}}\left[2 \int_{-\infty}^{\infty}\left(x^{2}-2 \sqrt{\alpha^{*}} x+|\alpha|^{2}+\alpha^{* 2}+\frac{2}{q-1}\right)^{\frac{1}{1-q}}\right. \\
& \otimes\left(x^{2}-2 \sqrt{\alpha} x+|\alpha|^{2}+\alpha^{2}+\frac{2}{q-1}\right)^{\frac{q}{1-q}} d x
\end{aligned}
$$




$$
\begin{aligned}
& +\frac{q}{1-q} \int_{-\infty}^{\infty}\left(x^{2}-2 \sqrt{\alpha^{*}} x+|\alpha|^{2}+\alpha^{* 2}+\frac{2}{q-1}\right)^{\frac{1}{1-q}}(2 x-2 \sqrt{2} \alpha)^{2} \\
& \left.\otimes\left(x^{2}-2 \sqrt{\alpha} x+|\alpha|^{2}+\alpha^{2}+\frac{2}{q-1}\right)^{\frac{2 q-1}{1-q}} d x\right]
\end{aligned}
$$

Applying again to (B.3), the result for $\left\langle p^{2}\right\rangle_{q}$ is

$$
\begin{aligned}
\left\langle p^{2}\right\rangle_{q}= & -\frac{A^{2}(q, \alpha)}{(1-q)}\left(\frac{q-1}{2}\right)^{\frac{2}{1-q}}\left\{2 \frac { \Gamma ( \frac { 3 + q } { q - 1 } ) } { \Gamma ( \frac { 2 q + 2 } { q - 1 } ) } \otimes F _ { D } \left(\frac{3+q}{q-1} ;\right.\right. \\
& \left.\frac{1}{q-1}, \frac{1}{q-1}, \frac{q}{q-1}, \frac{q}{q-1} ; \frac{2 q+2}{q-1} ; 1+\beta_{1}, 1+\beta_{2}, 1+\beta_{3}, 1+\beta_{4}\right) \\
& +\frac{q}{1-q}\left[8 \frac { \Gamma ( \frac { 3 + q } { q - 1 } ) } { \Gamma ( \frac { 4 q } { q - 1 } ) } \otimes F _ { D } \left(\frac{3+q}{q-1} ; \frac{1}{q-1}, \frac{1}{q-1}, \frac{2 q-1}{q-1}, \frac{2 q-1}{q-1}\right.\right. \\
& \left.\frac{4 q}{q-1} ; 1+\beta_{1}, 1+\beta_{2}, 1+\beta_{3}, 1+\beta_{4}\right) \\
& -8 \sqrt{2} \alpha \frac{\Gamma\left(\frac{2 q+2}{q-1}\right)}{\Gamma\left(\frac{4 q}{q-1}\right)} \otimes F_{D}\left(\frac{2 q+2}{q-1} ; \frac{1}{q-1}, \frac{1}{q-1}, \frac{2 q-1}{q-1}, \frac{2 q-1}{q-1}\right. \\
& \left.\frac{4 q}{q-1} ; 1+\beta_{1}, 1+\beta_{2}, 1+\beta_{3}, 1+\beta_{4}\right) \\
& +8 \alpha^{2} \frac{\Gamma\left(\frac{3 q+1}{q-1}\right)}{\Gamma\left(\frac{4 q}{q-1}\right)} \otimes F_{D}\left(\frac{3 q+1}{q-1} ; \frac{1}{q-1}, \frac{1}{q-1}, \frac{2 q-1}{q-1}, \frac{2 q-1}{q-1} ;\right. \\
& \left.\left.\left.; 1+\beta_{1}, 1+\beta_{2}, 1+\beta_{3}, 1+\beta_{4}\right)\right]\right\}
\end{aligned}
$$

\subsection{Mean value of $p$}

Analogously, we have for $\langle p\rangle_{q}$

$$
\begin{aligned}
\langle p\rangle_{q}= & -i A^{2}(q, \alpha)\left(\frac{q-1}{2}\right)^{\frac{2}{1-q}} \int_{-\infty}^{\infty}\left(x^{2}-2 \sqrt{\alpha^{*}} x+|\alpha|^{2}+\alpha^{* 2}+\frac{2}{q-1}\right)^{\frac{1}{1-q}} \\
& \otimes \frac{\partial}{\partial x}\left(x^{2}-2 \sqrt{\alpha} x+|\alpha|^{2}+\alpha^{2}+\frac{2}{q-1}\right)^{\frac{1}{1-q}} d x
\end{aligned}
$$


G. L. Ferri et al.

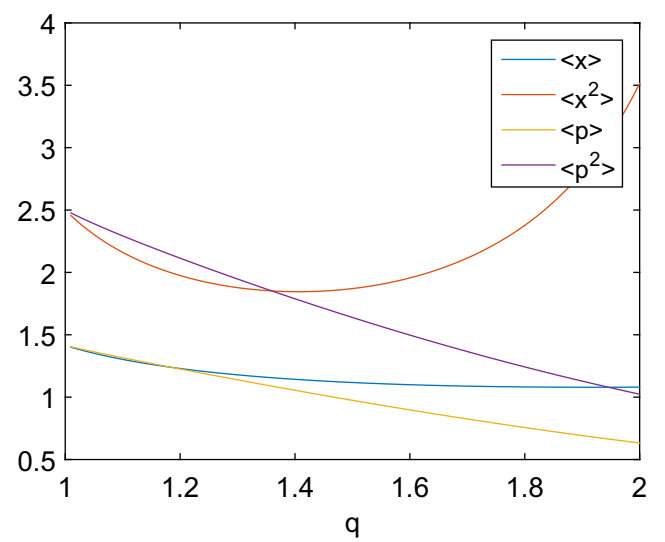

Fig. 1. Our four relevant mean values are plotted versus $q$.

or

$$
\begin{aligned}
\langle p\rangle_{q}= & -\frac{i A^{2}(q, \alpha)}{1-q}\left(\frac{q-1}{2}\right)^{\frac{2}{1-q}} \int_{-\infty}^{\infty}\left(x^{2}-2 \sqrt{\alpha^{*}} x+|\alpha|^{2}+\alpha^{* 2}+\frac{2}{q-1}\right)^{\frac{1}{1-q}} \\
& \otimes(2 x-2 \sqrt{2} \alpha)\left(x^{2}-2 \sqrt{\alpha} x+|\alpha|^{2}+\alpha^{2}+\frac{2}{q-1}\right)^{\frac{q}{1-q}} d x
\end{aligned}
$$

By recourse to (B.3) again, we obtain

$$
\begin{aligned}
\langle p\rangle_{q}= & -\frac{i A^{2}(q, \alpha)}{1-q}\left(\frac{q-1}{2}\right)^{\frac{2}{1-q}}\left[2 \frac { \Gamma ( \frac { 4 } { q - 1 } ) } { \Gamma ( \frac { 2 q + 2 } { q - 1 } ) } \otimes F _ { D } \left(\frac{4}{q-1} ;\right.\right. \\
& \left.\frac{1}{q-1}, \frac{1}{q-1}, \frac{q}{q-1}, \frac{q}{q-1} ; \frac{2 q+2}{q-1} ; 1+\beta_{1}, 1+\beta_{2}, 1+\beta_{3}, 1+\beta_{4}\right) \\
& -2 \sqrt{2} \alpha \frac{\Gamma\left(\frac{3+q}{q-1}\right)}{\Gamma\left(\frac{2 q+2}{q-1}\right)} \otimes F_{D}\left(\frac{3+q}{q-1} ; \frac{1}{q-1}, \frac{1}{q-1}, \frac{q}{q-1}, \frac{q}{q-1}\right. \\
& \left.\left.\frac{2 q+2}{q-1} ; 1+\beta_{1}, 1+\beta_{2}, 1+\beta_{3}, 1+\beta_{4}\right)\right]
\end{aligned}
$$

Figure 1 displays the $q$-dependence of our four relevant $q$-mean values. With the mean $q$-values obtained above, we can calculate $(\Delta x)_{q}(\Delta p)_{q}$. The uncertainties are plotted, as a function of $q$, in Fig. 2 .

\section{5. $\psi_{\alpha q}$ states form an over-complete basis}

It is easy to see that there is a one-to-one mapping $|\alpha\rangle \Leftrightarrow|\alpha, q\rangle$ that immediately arises from the well-known one-to-one mapping between $\mathrm{qEs}$ and ordinary ones. 


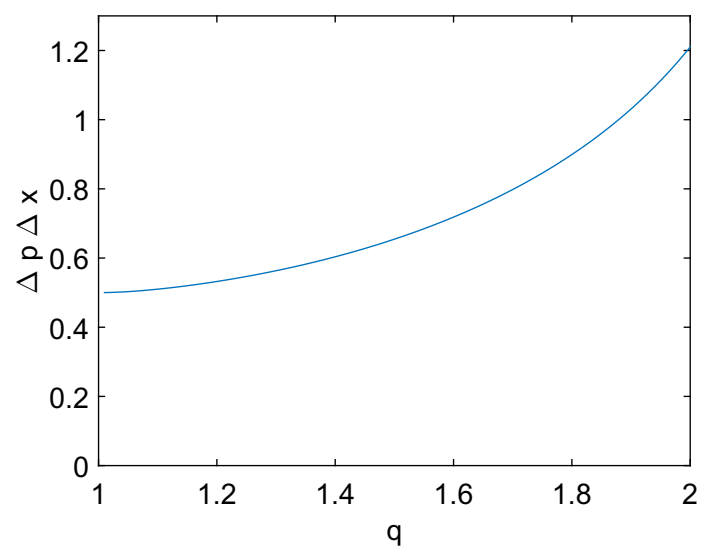

Fig. 2. Quantum uncertainties versus $q$.

This entails that one can write the unity operator as

$$
I=\int_{-\infty}^{\infty}|\alpha, q\rangle A(q, \alpha)\left\langle\alpha, q\left|d^{2} \alpha=\frac{1}{\pi} \int_{-\infty}^{\infty}\right| \alpha\right\rangle\langle\alpha| d^{2} \alpha
$$

with $A(q, \alpha)$ still an unknown constant. Here, $\lim _{q \rightarrow 1} A(q, \alpha)=\frac{1}{\pi}$.

Thus, for any $q$, the basis $\{|\alpha, q\rangle\}$ constitute an over-complete basis.

\section{Quantum Uncertainty in the Limit $q \rightarrow 1$}

We will show now that $\lim _{q \rightarrow 1}(\Delta x)_{q}(\Delta p)_{q}=\Delta x \Delta p=\frac{1}{2}$. This is to the essence in order to ensure that our $q$-extension of CS makes sense. For this endeavor, we use the approximation, for $q$ close to one, of the $\mathrm{qE}$. It is easily seen that one has

$$
[1+i(q-1) z]^{\frac{1}{1-q}}=\left[1-\frac{q-1}{2} z^{2}\right] e^{-i z}
$$

As a consequence of $(\underline{5.1})$, we obtain

$$
\begin{aligned}
{[1+} & \left.\frac{q-1}{2}\left(x^{2}-2 \sqrt{2} \alpha x+\alpha^{2}+|\alpha|^{2}\right)\right]^{\frac{1}{1-q}} \\
& =\left[1+\frac{q-1}{8}\left(x^{2}-2 \sqrt{2} \alpha x+\alpha^{2}+|\alpha|^{2}\right)^{2}\right] e^{-\frac{1}{2}\left(x^{2}-2 \sqrt{2} \alpha x+\alpha^{2}+|\alpha|^{2}\right)} \\
& =\left.\left(1+\frac{q-1}{2} \frac{\partial^{2}}{\partial \beta^{2}}\right) e^{-\frac{\beta}{2}\left(x^{2}-2 \sqrt{2} \alpha x+\alpha^{2}+|\alpha|^{2}\right)}\right|_{\beta=1} .
\end{aligned}
$$

The normalized $q$-CS reads, in this approximation,

$$
\psi_{\alpha q}(x)=\left.A^{-1}(q, \alpha)\left(1+\frac{q-1}{2} \frac{\partial^{2}}{\partial \beta^{2}}\right) e^{-\frac{\beta}{2}\left(x^{2}-2 \sqrt{2} \alpha x+\alpha^{2}+|\alpha|^{2}\right)}\right|_{\beta=1}
$$


Of course, $A(q, \alpha)$ needs evaluation. For this purpose, we calculate

$$
\begin{aligned}
A^{2}(q, \alpha)= & \left(1+\frac{q-1}{2} \frac{\partial^{2}}{\partial \beta^{2}}\right)\left(1+\frac{q-1}{2} \frac{\partial^{2}}{\partial \gamma^{2}}\right) \\
& \left.\otimes \int_{-\infty}^{\infty} e^{-\frac{\beta}{2}\left(x^{2}-2 \sqrt{2} \alpha^{*} x+\alpha^{* 2}+|\alpha|^{2}\right)} e^{-\frac{\gamma}{2}\left(x^{2}-2 \sqrt{2} \alpha x+\alpha^{2}+|\alpha|^{2}\right)} d x\right|_{\beta=\gamma=1} .
\end{aligned}
$$

By recourse to the Integral-Table result given in Ref. 31, we then find

$$
\begin{aligned}
A^{2}(q, \alpha)= & \sqrt{\pi}\left(1+\frac{q-1}{2} \frac{\partial^{2}}{\partial \beta^{2}}\right)\left(1+\frac{q-1}{2} \frac{\partial^{2}}{\partial \gamma^{2}}\right) \\
& \otimes\left[\frac{\sqrt{2}}{\sqrt{\beta+\gamma}} e^{-\frac{\beta}{2}\left(\alpha^{* 2}+|\alpha|^{2}\right)} e^{-\frac{\gamma}{2}\left(\alpha^{2}+|\alpha|^{2}\right)} e^{\frac{\left(\beta \alpha^{*}+\gamma \alpha\right)^{2}}{\beta+\gamma}}\right]_{\beta=\gamma=1} .
\end{aligned}
$$

We can thus write

$$
A^{2}(q, \alpha)=\sqrt{\pi}+(q-1) f_{1}(\alpha)+(q-1)^{2} f_{2}(\alpha),
$$

where $f_{1}$ and $f_{2}$ are nonsingular functions of $\alpha$. As a consequence,

$$
A(q, \alpha)=\sqrt{\sqrt{\pi}+(q-1) f_{1}(\alpha)+(q-1)^{2} f_{2}(\alpha)}
$$

and

$$
\lim _{q \rightarrow 1} A(q, \alpha)=\pi^{\frac{1}{4}}
$$

We can now write for $\left\langle x^{2}\right\rangle_{q}$

$$
\begin{aligned}
\left\langle x^{2}\right\rangle_{q}= & A^{-2}(q, \alpha)\left(1+\frac{q-1}{2} \frac{\partial^{2}}{\partial \beta^{2}}\right)\left(1+\frac{q-1}{2} \frac{\partial^{2}}{\partial \gamma^{2}}\right) \\
& \left.\otimes \int_{-\infty}^{\infty} e^{-\frac{\beta}{2}\left(x^{2}-2 \sqrt{2} \alpha^{*} x+\alpha^{* 2}+|\alpha|^{2}\right)} x^{2} e^{-\frac{\gamma}{2}\left(x^{2}-2 \sqrt{2} \alpha x+\alpha^{2}+|\alpha|^{2}\right)} d x\right|_{\beta=\gamma=1} .
\end{aligned}
$$

Using once more the Integral-Table,,$\underline{31}$ one has

$$
\begin{aligned}
& \left\langle x^{2}\right\rangle_{q} \\
& =A^{-2}(q, \alpha)\left(1+\frac{q-1}{2} \frac{\partial^{2}}{\partial \beta^{2}}\right)\left(1+\frac{q-1}{2} \frac{\partial^{2}}{\partial \gamma^{2}}\right) \\
& \otimes\left\{\frac{2^{\frac{3}{2}}}{(\beta+\gamma)^{\frac{3}{2}}} e^{-\frac{\beta}{2}\left(\alpha^{* 2}+|\alpha|^{2}\right)} e^{-\frac{\gamma}{2}\left(\alpha^{2}+|\alpha|^{2}\right)} e^{\frac{\left(\beta \alpha^{*}+\gamma \alpha\right)^{2}}{\beta+\gamma}}(2 i)^{-2} \sqrt{\pi} H_{2}\left[\frac{i\left(\beta \alpha^{*}+\gamma \alpha\right)}{\sqrt{\beta+\gamma}}\right]\right\} .
\end{aligned}
$$

As a consequence,

$$
\left\langle x^{2}\right\rangle_{q}=A^{-2}(q, \alpha)\left\{\sqrt{\pi}\left[\frac{\left(\alpha+\alpha^{*}\right)^{2}}{2}+\frac{1}{2}\right]+(q-1) g_{1}(\alpha)+(q-1)^{2} g_{2}(\alpha)\right\},
$$


where $g_{1}$ and $g_{2}$ are nonsingular functions of $\alpha$. Thus (see Appendix C),

$$
\lim _{q \rightarrow 1}\left\langle x^{2}\right\rangle_{q}=\frac{1}{2}+\frac{\left(\alpha+\alpha^{*}\right)^{2}}{2}=\left\langle x^{2}\right\rangle
$$

Proceeding now in similar fashion for $\langle x\rangle_{q}$ we obtain

$$
\begin{aligned}
\langle x\rangle_{q}= & A^{-2}(q, \alpha)\left(1+\frac{q-1}{2} \frac{\partial^{2}}{\partial \beta^{2}}\right)\left(1+\frac{q-1}{2} \frac{\partial^{2}}{\partial \gamma^{2}}\right) \\
& \left.\otimes \int_{-\infty}^{\infty} e^{-\frac{\beta}{2}\left(x^{2}-2 \sqrt{2} \alpha^{*} x+\alpha^{* 2}+|\alpha|^{2}\right)} x e^{-\frac{\gamma}{2}\left(x^{2}-2 \sqrt{2} \alpha x+\alpha^{2}+|\alpha|^{2}\right)} d x\right|_{\beta=\gamma=1} .
\end{aligned}
$$

According to the Integral-Table result, $\underline{31}$

$$
\begin{aligned}
\langle x\rangle_{q}= & A^{-2}(q, \alpha)\left(1+\frac{q-1}{2} \frac{\partial^{2}}{\partial \beta^{2}}\right)\left(1+\frac{q-1}{2} \frac{\partial^{2}}{\partial \gamma^{2}}\right) \\
& \otimes\left\{\frac{2}{\beta+\gamma} e^{-\frac{\beta}{2}\left(\alpha^{* 2}+|\alpha|^{2}\right)} e^{-\frac{\gamma}{2}\left(\alpha^{2}+|\alpha|^{2}\right)} e^{\frac{\left(\beta \alpha^{*}+\gamma \alpha\right)^{2}}{\beta+\gamma}}(2 i)^{-1} \sqrt{\pi} H_{1}\left[\frac{i\left(\beta \alpha^{*}+\gamma \alpha\right)}{\sqrt{\beta+\gamma}}\right]\right\},
\end{aligned}
$$

or

$$
\langle x\rangle_{q}=A^{-2}(q, \alpha)\left\{\sqrt{\pi}\left[\frac{\alpha+\alpha^{*}}{\sqrt{2}}\right]+(q-1) h_{1}(\alpha)+(q-1)^{2} h_{2}(\alpha)\right\},
$$

where $h_{1}$ and $h_{2}$ are again nonsingular functions of $\alpha$. Accordingly (see Appendix C),

$$
\lim _{q \rightarrow 1}\langle x\rangle_{q}=\frac{\alpha+\alpha^{*}}{\sqrt{2}}=\langle x\rangle .
$$

For $\left\langle p^{2}\right\rangle_{q}$, we have instead

$$
\begin{aligned}
\left\langle p^{2}\right\rangle_{q}= & -A^{-2}(q, \alpha)\left(1+\frac{q-1}{2} \frac{\partial^{2}}{\partial \beta^{2}}\right)\left(1+\frac{q-1}{2} \frac{\partial^{2}}{\partial \gamma^{2}}\right) \\
& \left.\otimes \int_{-\infty}^{\infty} e^{-\frac{\beta}{2}\left(x^{2}-2 \sqrt{2} \alpha^{*} x+\alpha^{* 2}+|\alpha|^{2}\right)} \frac{\partial^{2}}{\partial x^{2}} e^{-\frac{\gamma}{2}\left(x^{2}-2 \sqrt{2} \alpha x+\alpha^{2}+|\alpha|^{2}\right)} d x\right|_{\beta=\gamma=1}
\end{aligned}
$$

or

$$
\begin{aligned}
\left\langle p^{2}\right\rangle_{q}= & A^{-2}(q, \alpha)\left(1+\frac{q-1}{2} \frac{\partial^{2}}{\partial \beta^{2}}\right)\left(1+\frac{q-1}{2} \frac{\partial^{2}}{\partial \gamma^{2}}\right) \\
& \otimes \int_{-\infty}^{\infty} e^{-\frac{\beta}{2}\left(x^{2}-2 \sqrt{2} \alpha^{*} x+\alpha^{* 2}+|\alpha|^{2}\right)} \\
& \times\left.\left[\gamma^{2}(x-\sqrt{2} \alpha)^{2}-\gamma\right] e^{-\frac{\gamma}{2}\left(x^{2}-2 \sqrt{2} \alpha x+\alpha^{2}+|\alpha|^{2}\right)} d x\right|_{\beta=\gamma=1}
\end{aligned}
$$


As in previous cases, according to Integral-Table result, $\underline{31}$ we have

$$
\left\langle p^{2}\right\rangle_{q}=A^{-2}(q, \alpha)\left\{\sqrt{\pi}\left[\frac{1}{2}-\frac{\left(\alpha-\alpha^{*}\right)^{2}}{2}\right]+(q-1) k_{1}(\alpha)+(q-1)^{2} k_{2}(\alpha)\right\} .
$$

Here, $k_{1}$ and $k_{2}$ are nonsingular functions as well. Therefore, (see Appendix C),

$$
\lim _{q \rightarrow 1}\left\langle p^{2}\right\rangle_{q}=\frac{1}{2}-\frac{\left(\alpha-\alpha^{*}\right)^{2}}{2}=\left\langle p^{2}\right\rangle
$$

In analogy with the above case, we now also have

$$
\begin{aligned}
\langle p\rangle_{q}= & -i A^{-2}(q, \alpha)\left(1+\frac{q-1}{2} \frac{\partial^{2}}{\partial \beta^{2}}\right)\left(1+\frac{q-1}{2} \frac{\partial^{2}}{\partial \gamma^{2}}\right) \\
& \left.\otimes \int_{-\infty}^{\infty} e^{-\frac{\beta}{2}\left(x^{2}-2 \sqrt{2} \alpha^{*} x+\alpha^{* 2}+|\alpha|^{2}\right)} \frac{\partial}{\partial x} e^{-\frac{\gamma}{2}\left(x^{2}-2 \sqrt{2} \alpha x+\alpha^{2}+|\alpha|^{2}\right)} d x\right|_{\beta=\gamma=1}
\end{aligned}
$$

and, after employing again the Integral-Table result,,$\frac{31}{}$

$$
\langle p\rangle_{q}=-i A^{-2}(q, \alpha)\left\{\sqrt{\pi}\left[\frac{\alpha-\alpha^{*}}{\sqrt{2}}\right]+(q-1) l_{1}(\alpha)+(q-1)^{2} l_{2}(\alpha)\right\},
$$

where $l_{1}$ and $l_{2}$ are nonsingular functions of $\alpha$. Thus, (see Appendix C),

$$
\lim _{q \rightarrow 1}\langle p\rangle_{q}=\frac{\alpha-\alpha^{*}}{i \sqrt{2}}=\langle p\rangle .
$$

From $(\underline{5.12)}, \underline{(5.16)}, \underline{(5.20)}$ and $\underline{(5.23)}$, we obtain

$$
\lim _{q \rightarrow 1}(\Delta x)_{q}(\Delta p)_{q}=\Delta x \Delta p=\frac{1}{2} .
$$

For the $q$-distribution, with $q$ close to 1 , and using

$$
|q, \alpha\rangle=\left.A^{-1}(q, \alpha)\left(1+\frac{q-1}{2} \frac{\partial^{2}}{\partial \beta^{2}}\right) \int_{-\infty}^{\infty} e^{-\frac{\beta}{2}\left(x^{2}-2 \sqrt{2} \alpha^{*} x+\alpha^{* 2}+|\alpha|^{2}\right)}|x\rangle d x\right|_{\beta=1},
$$

we have

$$
\begin{aligned}
\langle k \mid q, \alpha\rangle= & \frac{A^{-1}(q, \alpha)}{\sqrt{2 \pi}}\left(1+\frac{q-1}{2} \frac{\partial^{2}}{\partial \beta^{2}}\right) \\
& \left.\otimes \int_{-\infty}^{\infty} e^{-i k x} e^{-\frac{\beta}{2}\left(x^{2}-2 \sqrt{2} \alpha x+\alpha^{2}+|\alpha|^{2}\right)} d x\right|_{\beta=1} .
\end{aligned}
$$

Again, from the Integral-Table result, $\frac{31}{1}$ we can write

$$
\langle k \mid q, \alpha\rangle=A^{-1}(q, \alpha)\left[e^{-\frac{1}{2}\left(k^{2}+2 \sqrt{2} i \alpha k-\alpha^{2}+|\alpha|^{2}\right)}+(q-1) f(\alpha, k)\right],
$$

where $f$ is nonsingular. Using the results given there, we have

$$
\lim _{q \rightarrow 1}\langle k \mid q, \alpha\rangle=\pi^{-\frac{1}{4}} e^{-\frac{1}{2}\left(k^{2}+2 \sqrt{2} i \alpha k-\alpha^{2}+|\alpha|^{2}\right)}=\langle k \mid \alpha\rangle,
$$


and as a consequence,

$$
\lim _{q \rightarrow 1}|\langle k \mid q, \alpha\rangle|^{2}=|\langle k \mid \alpha\rangle|^{2}=\pi^{-\frac{1}{2}} e^{-(k-p)^{2}},
$$

a nice result indeed!

\section{Conclusions}

We have introduced and studied in this work special $q$-states that one might denominate Tsallis' pseudo-coherent ones (that have nothing to do with the so-called $q$-CS of Quesne, Eremin-Meldianov and others ${ }^{22}$ ).

Also, we obtained some interesting preliminary results. In particular, we have exhibited the $q$-dependence of the quantum uncertainty, that is minimal for $q=1$. We emphasize that we have obtained the first over-complete basis of Tsallis literature. This should be an interesting addition to such body of work. Summing up:

- We determined the most important relationships governing the new Tsallis' pseudo-CS.

- In particular, let us reiterate, we find that, in the limit $q \rightarrow 1$, minimal uncertainty is attained (for $q=1$ ), which constitutes a fundamental result.

- We saw that the Tsallis' pseudo-CS constitute an over-complete basis for any $q$.

\section{Appendix A. Proof of Eq. (2.9)}

It is very well known that the annihilation operator for the one-dimensional HO is given by

$$
\hat{a}=\frac{\hat{x}+i \hat{p}}{\sqrt{2}} .
$$

In the $x$-representation of Quantum Mechanics, this operator is expressed via

$$
\hat{a}(x)=\frac{1}{\sqrt{2}}\left(x+\frac{d}{d x}\right) .
$$

Thus, a CS is defined as the eigenfunction

$$
\hat{a}(x) \psi_{\alpha}(x)=\frac{1}{\sqrt{2}}\left(x \psi_{\alpha}(x)+\frac{d \psi_{\alpha}(x)}{d x}\right)=\alpha \psi_{\alpha}(x),
$$

or, equivalently,

$$
\frac{d \psi_{\alpha}(x)}{d x}=(\sqrt{2} \alpha-x) \psi_{\alpha}(x)
$$

The solution of (A.4) is

$$
\psi_{\alpha}(x)=C e^{-\frac{x^{2}}{2}} e^{\sqrt{2} \alpha x} .
$$

The constant $C$ can be evaluated using the normalization condition

$$
\int_{-\infty}^{\infty}\left|\psi_{\alpha}(x)\right|^{2} d x=|C|^{2} \int_{-\infty}^{\infty} e^{-x^{2}} e^{\sqrt{2}\left(\alpha+\alpha^{*}\right) x} d x=1 .
$$


Accordingly,

$$
\int_{-\infty}^{\infty}\left|\psi_{\alpha}(x)\right|^{2} d x=|C|^{2} e^{\frac{\left(\alpha+\alpha^{*}\right)^{2}}{2}} \int_{-\infty}^{\infty} e^{-\left(x-\frac{\alpha+\alpha^{*}}{\sqrt{2}}\right)^{2}} d x=1 .
$$

By recourse to the result given in the Table,,$\underline{31}$ we now obtain

$$
\int_{-\infty}^{\infty} e^{-\left(x-\frac{\alpha+\alpha^{*}}{\sqrt{2}}\right)^{2}} d x=\sqrt{\pi}
$$

As a consequence,

$$
C=\pi^{-\frac{1}{4}} e^{-\frac{\left(\alpha+\alpha^{*}\right)^{2}}{4}}
$$

Thus, we have for $\psi_{\alpha}(x)$ the expression

$$
\psi_{\alpha}(x)=\pi^{-\frac{1}{4}} e^{-\frac{\left(\alpha+\alpha^{*}\right)^{2}}{4}} e^{-\frac{x^{2}}{2}} e^{\sqrt{2} \alpha x},
$$

or, equivalently,

$$
\psi_{\alpha}(x)=e^{i \alpha_{R} \alpha_{I}} \pi^{-\frac{1}{4}} e^{-\frac{\alpha^{2}}{2}} e^{-\frac{|\alpha|^{2}}{2}} e^{-\frac{x^{2}}{2}} e^{\sqrt{2} \alpha x},
$$

where $\alpha=\alpha_{R}+i \alpha_{I}$. As $e^{i \alpha_{R} \alpha_{I}}$ is an imaginary phase, it can be eliminated from (A.11) to finally obtain

$$
\psi_{\alpha}(x)=\pi^{-\frac{1}{4}} e^{-\frac{\alpha^{2}}{2}} e^{-\frac{|\alpha|^{2}}{2}} e^{-\frac{x^{2}}{2}} e^{\sqrt{2} \alpha x} .
$$

\section{Appendix B. Lauricella Functions}

Lauricella functions $F$ can be regarded as generalizations to several variables of the Gauss hypergeometric functions. They were investigated at the end of the 19th century by Giuseppe Lauricella (18671913), an Italian mathematician mostly known by his contribution to elasticity theory. The fourth Lauricella function of four variables is given by $\underline{32}$

$$
\begin{aligned}
& F_{D}\left(a ; b_{1}, b_{2}, b_{3}, b_{4} ; c ; x_{1}, x_{2}, x_{3}, x_{4}\right) \\
& =\sum_{m_{1}=0}^{\infty} \sum_{m_{2}=0}^{\infty} \sum_{m_{3}=0}^{\infty} \sum_{m_{4}=0}^{\infty} \frac{(a)_{m_{1}+m_{2}+m_{3}+m_{4}}\left(b_{1}\right)_{m_{1}}\left(b_{2}\right)_{m_{2}}\left(b_{3}\right)_{m_{3}}\left(b_{4}\right)_{m_{4}}}{(c)_{m_{1}+m_{2}+m_{3}+m_{4}}} \\
& \otimes \frac{x_{1}^{m_{1}} x_{2}^{m_{2}} x_{3}^{m_{3}} x_{4}^{m_{4}}}{m_{1} ! m_{2} ! m_{3} ! m_{4} !}
\end{aligned}
$$

This function satisfies 32

$$
\begin{gathered}
\int_{0}^{1} u^{a-1}(1-u)^{c-a-1}\left(1-u x_{1}\right)^{-b_{1}}\left(1-u x_{2}\right)^{-b_{2}}\left(1-u x_{3}\right)^{-b_{3}}\left(1-u x_{4}\right)^{-b_{4}} d u \\
=\frac{\Gamma(a) \Gamma(c-a)}{\Gamma(c)} F_{D}\left(a ; b_{1}, b_{2}, b_{3}, b_{4} ; c ; x_{1}, x_{2}, x_{3}, x_{4}\right)
\end{gathered}
$$


After two variable changes, we can deduce, from (B.2), the relation

$$
\begin{gathered}
\int_{0}^{\infty} u^{c-a-1}(1-u)^{b_{1}+b_{2}+b_{3}+b_{4}-c}\left(u_{1}+z_{1}\right)^{-b_{1}}\left(u_{2}+z_{2}\right)^{-b_{2}}\left(u_{3}+z_{3}\right)^{-b_{3}}\left(u_{4}+z_{4}\right)^{-b_{4}} d u \\
\quad=\frac{\Gamma(a) \Gamma(c-a)}{\Gamma(c)} F_{D}\left(a ; b_{1}, b_{2}, b_{3}, b_{4} ; c ; 1-z_{1}, 1-z_{2}, 1-z_{3}, 1-z_{4}\right) .
\end{gathered}
$$

\section{Appendix C. Reviewing Uncertainty Relations for CS}

For the sake of completeness, we give here some well-known results that are needed in determining uncertainties. For an ordinary $\operatorname{CS}|\alpha\rangle$, we have

$$
\left\langle x^{2}\right\rangle=\pi^{-\frac{1}{2}} \int_{-\infty}^{\infty} e^{-\frac{1}{2}\left(x^{2}-2 \sqrt{2} \alpha^{*} x+\alpha^{* 2}+|\alpha|^{2}\right)} x^{2} e^{-\frac{1}{2}\left(x^{2}-2 \sqrt{2} \alpha x+\alpha^{2}+|\alpha|^{2}\right)} d x .
$$

With the use of the Integral-Table result, $\underline{31}$ we find

$$
\left\langle x^{2}\right\rangle=(2 i)^{-2} H_{2}\left[\frac{i\left(\alpha^{*}+\alpha\right)}{\sqrt{2}}\right]
$$

and then

$$
\left\langle x^{2}\right\rangle=\frac{1}{2}+\frac{\left(\alpha+\alpha^{*}\right)^{2}}{2} .
$$

For $\langle x\rangle$ the situation is quite similar

$$
\langle x\rangle=\pi^{-\frac{1}{2}} \int_{-\infty}^{\infty} e^{-\frac{1}{2}\left(x^{2}-2 \sqrt{2} \alpha^{*} x+\alpha^{* 2}+|\alpha|^{2}\right)} x e^{-\frac{1}{2}\left(x^{2}-2 \sqrt{2} \alpha x+\alpha^{2}+|\alpha|^{2}\right)} d x .
$$

Using the Integral-Table result $\underline{31}$ again, we obtain

$$
\langle x\rangle=(2 i)^{-1} H_{1}\left[\frac{i\left(\alpha^{*}+\alpha\right)}{\sqrt{2}}\right]
$$

and thus

$$
\langle x\rangle=\frac{\alpha+\alpha^{*}}{\sqrt{2}} .
$$

For $\langle p\rangle$, the integral is somewhat more complicated

$$
\left\langle p^{2}\right\rangle=-\pi^{-\frac{1}{2}} \int_{-\infty}^{\infty} e^{-\frac{1}{2}\left(x^{2}-2 \sqrt{2} \alpha^{*} x+\alpha^{* 2}+|\alpha|^{2}\right)} \frac{\partial^{2}}{\partial x^{2}} e^{-\frac{1}{2}\left(x^{2}-2 \sqrt{2} \alpha x+\alpha^{2}+|\alpha|^{2}\right)} d x
$$

or

$$
\left\langle p^{2}\right\rangle=\pi^{-\frac{1}{2}} \int_{-\infty}^{\infty} e^{-\frac{1}{2}\left(x^{2}-2 \sqrt{2} \alpha^{*} x+\alpha^{* 2}+|\alpha|^{2}\right)}\left[1-(x-\sqrt{2} \alpha)^{2}\right] e^{-\frac{1}{2}\left(x^{2}-2 \sqrt{2} \alpha x+\alpha^{2}+|\alpha|^{2}\right)} d x .
$$

Now, by recourse to the Integral-Table result, $\frac{31}{}$ we obtain

$$
\left\langle p^{2}\right\rangle=1-2 \alpha^{2}-i \sqrt{2} \alpha H_{1}\left[\frac{i\left(\alpha^{*}+\alpha\right)}{\sqrt{2}}\right]+\frac{1}{4} H_{2}\left[\frac{i\left(\alpha^{*}+\alpha\right)}{\sqrt{2}}\right]
$$


or

$$
\left\langle p^{2}\right\rangle=\frac{1}{2}-\frac{\left(\alpha-\alpha^{*}\right)^{2}}{2} .
$$

For dealing with $\langle p\rangle$, one starts with

$$
\langle p\rangle=-i \pi^{-\frac{1}{2}} \int_{-\infty}^{\infty} e^{-\frac{1}{2}\left(x^{2}-2 \sqrt{2} \alpha^{*} x+\alpha^{* 2}+|\alpha|^{2}\right)} \frac{\partial}{\partial x} e^{-\frac{1}{2}\left(x^{2}-2 \sqrt{2} \alpha x+\alpha^{2}+|\alpha|^{2}\right)} d x
$$

or

$$
\langle p\rangle=i \pi^{-\frac{1}{2}} \int_{-\infty}^{\infty} e^{-\frac{1}{2}\left(x^{2}-2 \sqrt{2} \alpha^{*} x+\alpha^{* 2}+|\alpha|^{2}\right)}(x-\sqrt{2} \alpha) e^{-\frac{1}{2}\left(x^{2}-2 \sqrt{2} \alpha x+\alpha^{2}+|\alpha|^{2}\right)} d x
$$

and, finally,

$$
\langle p\rangle=\frac{\alpha-\alpha^{*}}{i \sqrt{2}} .
$$

Accordingly, the well-known uncertainty relation for a CS becomes

$$
\Delta x \Delta p=\frac{1}{2},
$$

i.e., minimal uncertainty, the main feature of CS.

\section{References}

1. C. Tsallis, J. Stat. Phys. 52, 479 (1988).

2. C. Tsallis, Introduction to Nonextensive Statistical Mechanics: Approaching a Complex World (Springer, NY, 2009).

3. A. Adare et al., Phys. Rev. D 83, 052004 (2011).

4. G. Wilk and Z. Wlodarczyk, Physica A 305, 227 (2002).

5. R. M. Pickup et al., Phys. Rev. Lett. 102, 097202 (2009).

6. E. Lutz and F. Renzoni, Nat. Phys. 9, 615 (2013).

7. R. G. DeVoe, Phys. Rev. Lett. 102, 063001 (2009).

8. Z. Huang et al., J. Stat. Mech. L050015 (2010).

9. J. Prehl, C. Essex and K. H. Hoffman, Entropy 14, 701 (2012).

10. B. Liu and J. Goree, Phys. Rev. Lett. 100, 055003 (2018).

11. O. Afsar and U. Tirnakli, Europhys. Lett. 101, 20003 (2013).

12. U. Tirnakli, C. Tsallis and C. Beck, Phys. Rev. E 79, 056209 (2009).

13. G. Ruiz, T. Bountis and C. Tsallis, Int. J. Bifurcation Chaos 22, 1250208 (2012).

14. C. Beck and S. Miah, Phys. Rev. E 87, 031002 (2013).

15. H. J. Carmichael, Statistical Methods in Quantum Optics: Master Equations and Fokker-Planck Equations, Texts and Monographs in Physics (Springer, Berlin, 1998).

16. G. Wilk and Z. Wlodarczyk, Phys. Rev. Lett. 84, 2770 (2000).

17. J. Rozynek and G. Wilk, J. Phys. G Nucl. Part. Phys. 36, 125108 (2009).

18. C. M. Gell-Mann and C. Tsallis, Nonextensive Entropy: Interdisciplinary Applications (Oxford University Press, New York, 2004).

19. S. Abe, Astrophys. Space Sci. 305, 241 (2006).

20. S. Picoli et al., Braz. J. Phys. 39, 468 (2009).

21. F. D. Nobre, M. A. Rego-Monteiro and C. Tsallis, Phys. Rev. Lett. 106, 140601 (2011).

22. C. Quesne, arXiv:quant-ph/0206188; V. V. Eremin and A. A. Meldianov, arXiv:quant-ph/0810.1967. 
23. R. J. Glauber, Phys. Rev. Lett. 10, 84 (1963).

24. R. J. Glauber, Phys. Rev. 130, 2529 (1963).

25. R. J. Glauber, Phys. Rev. 131, 493 (1963).

26. R. J. Glauber, Quantum Optics and Electronics, eds. C. deWitt, A. Blandin and C. Cohen-Tannoudji (Gordon and Breach, New York, 1965).

27. I. S. Gradshteyn and I. M. Ryzhik, Table of Integrals, Series and Products, Vol. 7.374 (Academic Press, New York, 1965), p. 837.

28. I. S. Gradshteyn and I. M. Ryzhik, Table of Integrals, Series and Products, Vol. 3.462 (Academic Press, New York, 1965), p. 337.

29. H. J. Carmichael, Statistical Methods in Quantum Optics I: Master Equations and Fokker-Planck Equations (Springer-Verlag, Berlin, Heidelberg, 1999).

30. I. S. Gradshteyn and I. M. Ryzhik, Table of Integrals, Series and Products, Vol. 3.384 (Academic Press, New York, 1965), p. 320.

31. I. S. Gradshteyn and I. M. Ryzhik, Table of Integrals, Series and Products, Vol. 3.462 (Academic Press, New York, 1965), p. 338.

32. A. M. Mathai, H. J. Haubold, Special Functions for Applied Scientists (Springer, Berlin, 2008). 